\title{
Contribution à l'étude de la résistance à la sécheresse chez le blé tendre (Triticum aesti- vum L.) et chez le blé dur (Triticum durum Desf.) : étude de l'accumulation de la proline au cours du cycle de développement
}

Philippe MONNEVEUX $\left({ }^{1}\right)$ \& Mahdi NEMMAR $\left({ }^{2}\right)$

Ecole Nationale Supérieure Agronomique, Chaire de Phytotechnie et I.N.R.A., Station d'Amélioration des Plantes, 9, place Viala, 34060 Montpellier Cedex

L'évolution de la teneur en proline des feuilles a été suivie, chez le blé tendre et chez le blé dur, au long du cycle de développement, au cours de trois années.

L'étude montre que la teneur en proline est très étroitement liée à la température maximale journalière et à la pluviométrie, l'accumulation de l'acide aminé intervenant comme une réponse au déficit hydrique.

Un classement des variétés étudiées a pu être établi sur la base de la teneur maximale : les variétés caractérisées par les accumulations les plus importantes sont des variétés connues pour leur résistance élevée à la sécheresse, les variétés de blé dur ont des teneurs plus élevées que les variétés de blé tendre. Le classement des variétés au sein de chaque espèce est proche de celui obtenu à partir de la mesure de la résistance foliaire à la transpiration lors du palier hydrique.

L'accumulation de la proline qui nous semble jouer un rôle important comme mécanisme biochimique de résistance au déficit hydrique pourrait être utilisée dans la recherche de géniteurs de résistance.

Mots clés additionnels : Teneur en proline, résistance foliaire à la transpiration.

Contribution to the study of biochemical mechanisms of resistance to water stress: proline accumulation during the vegetative cycle of bread wheat (Triticum aestivum L.) and durum wheat (Triticum durum Desf.).

Leaf proline values were followed, in bread wheat and durum wheat, during the development cycle and over three years. Proline value was very closed linked to maximal day temperature and to rainfall, accumulation of the aminoacid behaving as a response to water deficit. The varieties tested were classified on the basis of their maximal proline value : varieties with the highest values are known for their high resistance to drought ; durum wheat varieties have higher values than bread wheat varieties. The classification of varieties within each species close to that obtained by measurement of foliar resistance to transpiration. Proline accumulation, which seems have an important role as a biochemical mechanism of resistance to water stress, should be used in the search for resistant parents.

Additional key words : Proline value, foliar resistance to transpiration.

(') Adresse actuelle : Institut National de Développement Rural, Section Agronomie, B.P. A296, R.P. Thies (Sénégal). $\left({ }^{2}\right)$ Adresse actuelle : Institut des Grandes Cultures, El Harrach, Alger X (Algérie). 


\section{INTRODUCTION}

La sécheresse est l'un des tout premiers facteurs intervenant dans la limitation des rendements; elle affecte fortement la production des zones arides et semi-arides, caractérisées par des pluies rares et irrégulières et par des températures souvent élevées.

Chez les céréales, l'amélioration des techniques culturales, en particulier l'introduction de l'irrigation, permet de réduire les effets des déficits hydriques. En l'absence d'irrigation, l'amélioration génétique reste sans doute le moyen le plus efficace pour disposer d'un matériel végétal susceptible d'assurer une production satisfaisante.

Celle-ci peut conduire, selon MAY \& MilthorPe (1962), à l'obtention :

- soit de variétés capables d'《échapper » à la sécheresse intervenant lors du remplissage et de la maturation du grain, en d'autres termes, capables d'éviter l'échaudage consécutif à l'action du manque d'eau et des hautes températures en fin de cycle ;

- soit de variétés capables de "tolérer " l'action de la sécheresse : cette tolérance correspond à une capacité de la plante à maintenir ses activités métaboliques malgré le déficit hydrique et à les reprendre de façon normale après ce déficit ;

- soit de variétés capables de « résister » à la sécheresse par le maintien d'un potentiel hydrique intrinsèque important. Cette " résistance constitutionnelle " (STOCKER, 1961), serait liée au rôle conjugué et souvent complémentaire de facteurs morphologiques ou biochimiques intervenant au niveau de la plante entière et au niveau cellulaire.

$\mathrm{Au}$ niveau de la plante entière, certains facteurs peuvent :

- limiter les pertes en eau : présence de barbes chez les céréales qui, d'après GRIGNAC (1965), expliquerait en partie la supériorité des blés durs sur les blés tendres mutiques quant à la résistance à la sécheresse, présence de stomates nombreux et de petite taille (PARCEVAUX, 1963), à fermeture rapide (PARKER, 1972) ;

- favoriser l'alimentation en eau : système racinaire développé et profond, racines nombreuses et divisées ; le rapport pondéral racines/parties aériennes est généralement plus élevé chez les variétés de céréales résistantes à la sécheresse (SANDHU \& LAUDE, 1958).

$\mathrm{Au}$ niveau cellulaire, le maintien d'une pression osmotique interne élevée est liée à la taille et à la viscosité protoplasmique de la cellule, à l'épaisseur des parois cellulaires (MAY \& MILTHORPE, 1962) et à l'accumulation de certains acides aminés ; parmi ceuxci, la proline semble jouer un rôle de première importance: son accumulation rapide lors d'un déficit hydrique a été mise en évidence chez de nombreuses plantes, en particulier chez l'orge par SAVITSKAYA (1967) et chez le blé par TYANKOVA (1967) et par VLASYUK et al. (1968). La proline serait synthétisée à partir de l'acide glutamique (fig. 1) par l'intermédiaire de l'acide semialdéhyde glutamique et de l'acide 5carboxylique $\Delta 1$ pyrroline (P5C) ; le déficit hydrique interviendrait, d'après BOGGESS \& STEWART (1976), principalement sur la formation du P5C, plus que sur

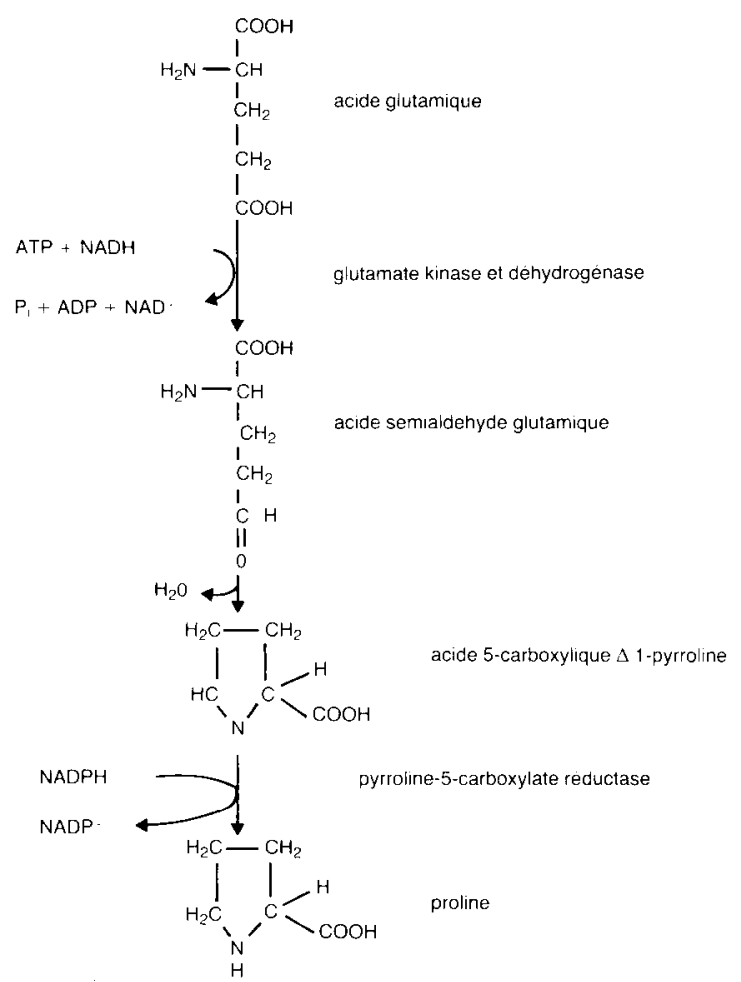

Figure 1

Synthèse de la proline, d'après Lehninger (1975).

Proline synthesis, after Lehninger (1975).

la réduction de cet intermédiaire en proline. L'accumulation de proline consécutive à la sécheresse serait à la fois le résultat d'une diminution de la synthèse protéique (STEWART \& BOGGESS, 1978), d'une inactivation des réactions d'oxydation conduisant à la formation d'acide glutamique (STEWART et al., 1977), et d'une reconversion en proline des produits d'oxydation (STEWART \& BOGGESS, 1978).

SiNGH et al. (1973a) ont noté, chez les céréales soumises à l'action de la sécheresse, des accumulations de proline d'autant plus importantes que les génotypes sont plus résistants ; SiNGH et al. (1973b) notent, chez l'orge, que cette accumulation a lieu principalement au niveau des limbes foliaires et, à un degré moindre, au niveau des racines et des apex. Chez le blé, l'augmentation des teneurs en proline et en arginine des feuilles serait, selon ProtsEnKo et al. (1968), un des symptômes de l'adaptation à la sécheresse, les variétés résistantes accumulant ces 2 acides aminés en quantités plus importantes.

Le rôle de la proline dans la résistance à la sécheresse est toutefois encore mal connu : on ignore par exemple si l'accumulation est un simple symptôme de l'action de la sécheresse (SINGH et al., 1973b) ou si elle constitue un véritable « mécanisme de résistance » (HUBAC \& GUERRIER, 1972) : la proline pourrait intervenir dans ce cas en régulant, par l'augmentation de sa concentration, la pression osmotique interne (STEWART \& LEE, 1974) mais aussi en inhibant les mécanismes d'auxesis (HUBAC \& GUERRIER, 1972) ou en constituant un « stock d'azote » utilisable par la plante postérieurement à la période de souffrance hydrique (PAQUIN, 1977 ; TALL \& ROSENTAL, 1979). 
Le but de la présente étude, a été de suivre, au cours de 3 campagnes, l'évolution de la teneur en proline des feuilles de blé tendre et de blé dur, en cherchant :

- à relier l'accumulation aux stades de développement de la plante et à certaines caractéristiques climatiques (pluviométrie, températures maximales journalières), ceci afin de préciser si l'accumulation est un phénomène intrinsèque lié au développement, ou si, au contraire, elle dépend essentiellement des variations des conditions de milieu (température, humidité) ;

- à mettre en évidence d'éventuelles différences génotypiques chez des variétés de blé tendre et de blé dur dans l'aptitude à accumuler la proline et à voir si, dans ce cas, les différences observées peuvent être liées à des niveaux différents de résistance à la sécheresse de ces variétés : dans ce cadre, les résultats obtenus ont été comparés, dans l'un des essais, à des mesures de résistance foliaire à la transpiration effectuées sur les mêmes variétés.

\section{MATÉRIEL ET MÉTHODE}

\section{A. Mise en place des essais}

Les essais ont été menés au cours de 3 années (1980, 1981, 1983).

Les mesures et analyses ont été effectuées sur du matériel végétal cultivé au champ, en conditions naturelles ; les pluviométries journalières et les températures maximales journalières ont été enregistrées au cours des 3 essais.

Les variétés retenues pour les expérimentations sont cultivées au Domaine I.N.R.A. de Lavalette (1980 et 1981), et dans les parcelles expérimentales de l'E.N.S.A. de Montpellier (1983) ; chaque variété est semée sur 5 lignes de $1,50 \mathrm{~m}$ (distance de $25 \mathrm{~cm}$ entre les lignes).

Une fumure de fond $\left(100 \mathrm{u} . \mathrm{P}_{2} \mathrm{O}_{5}, 100 \mathrm{u} . \mathrm{K}_{2} \mathrm{O}\right)$ est apportée, ainsi qu'une fumure azotée fractionnée en 2 fois, à la levée $(50 \mathrm{u}$.) et au tallage $(50 \mathrm{u}$.).

\section{B. Choix des variétés}

Les tableaux 1 et 2 donnent la liste des variétés testées au cours des 3 essais ; parmi les variétés de blé tendre testées, 10 sont présentes dans tous les essais.

\section{Dosage de la proline}

Le dosage est effectué sur le tiers médian de la dernière feuille.

La méthode utilisée est celle de TROLL 8 LINDSLEY (1955), modifiée par DREIER \& GÖRING (1974) : la proline est extraite à $85^{\circ} \mathrm{C}$ par le méthanol et colorée par la ninhydrine, en présence d'acide acétique et d'acide orthophosphorique : la mesure de la coloration rouge obtenue est effectuée sur un spectrophotomètre PERKIN-ELMER 55E à $528 \mathrm{~nm}$.
TABLEAU 1

Variétés de blé tendre utilisées dans les essais. Bread wheat varieties used in the experiments.

Variétés témoins (présentes dans tous les essais) :

Biezostaia, Cheyenne, Ducat, Etoile de Choisy, Florence $\times$ Aurore, Maris Hobbitt, Lutin, Roazon, Talent, Top.

Autres variétés testées (et origine des variétés) :

\begin{tabular}{ll}
\hline \hline \multicolumn{1}{c}{\begin{tabular}{c} 
Essai $\mathrm{n}^{\circ} 2$ \\
\multicolumn{1}{c}{1981}
\end{tabular}} & \multicolumn{1}{c}{$\begin{array}{c}\text { Essai } \mathrm{n}^{\circ} 3 \\
1983\end{array}$} \\
\hline Alass (Syrie) & Anza (Mexique) \\
Arvand (Syrie) & Arz (Liban) \\
Arz (Liban) & Champlein (France) \\
Banni (Yemen) & Inia 66 (Mexique) \\
Capitole (France) & Mahon Demias (Algérie) \\
Hamsi (Yemen) & Mexicano (Mexique) \\
Haramoun (Syrie) & Pumaflor (Algérie) \\
Inia 66 (Mexique) & Siete Cerros (Mexique) \\
Khachabi (Yemen) & Strampelli (Italie) \\
Mahon Demias (Algérie) & \\
Maris Huntsman (Gd $*$ Bretagne) & \\
Mexicano (Mexique) & \\
Odwari (Yemen) & \\
Pumaflor (Algérie) & \\
Siete Cerros (Mexique) & \\
\hline \hline
\end{tabular}

TABLEAU 2

Variétés de blé dur utilisées dans les essais.

Durum wheat varieties used in the experiments.

\begin{tabular}{|c|c|c|}
\hline Essai $n^{\circ} 1$ & Essaj $n^{\circ} 2$ & Essai $n^{\circ} 3$ \\
\hline Agathe (France) & Bidi 17 (Algérie) & $\begin{array}{c}\text { Gloire de Montgolfier } \\
\text { (Algérie) }\end{array}$ \\
\hline Bidi 17 (Algérie) & $\begin{array}{c}\text { Gloire de Montgolfier } \\
\text { (Algérie) }\end{array}$ & Inrat 69 (Tunisie) \\
\hline Capeiti (Italie) & Haurani (Liban) & $\begin{array}{l}\text { Mohamed Ben Bachir } \\
\text { (Algérie) }\end{array}$ \\
\hline Cocorit (Mexique) & Inrat 69 (Tunisie) & Montpellier (Algérie) \\
\hline Créso (Italie) & $\begin{array}{c}\text { Mohamed Ben Bachir } \\
\text { (Algérie) }\end{array}$ & Oued Zenati (Algérie) \\
\hline Durtal (France) & Montpellier (Algérie) & $\begin{array}{c}\text { Polonicum } \times \text { Zenati } \\
\text { (Algérie) }\end{array}$ \\
\hline Lakota (U.S.A.) & $\begin{array}{c}\text { Polonicum } \times \text { Zenati } \\
\text { (Algérie) }\end{array}$ & \\
\hline $\begin{array}{l}\text { Mondur (France) } \\
\text { Tomclair (France) } \\
\text { Valnova (Italie) }\end{array}$ & & \\
\hline
\end{tabular}

\section{Mesure de la résistance foliaire à la transpiration}

Sur l'essai $n^{\circ} 3$ (1983), ont été effectuées des mesures de résistance foliaire à la diffusion de vapeur d'eau ou résistance foliaire à la transpiration (R.F.T.) à l'aide d'un poromètre à diffusion à fente étroite LAMBDA LI - 65, selon la méthode de BANGE (1953).

Les mesures sont pratiquées sur la partie médiane de la face abaxiale de l'avant-dernière feuille bien développée, lors du palier hydrique, qui correspond à une période particulière de sensibilité à la sécheresse chez le blé. Les résultats sont exprimés $\mathrm{s} / \mathrm{cm}$. 


\section{RÉSULTATS}

\section{A. Comparaison des essais}

La comparaison des courbes d'accumulation obtenues au cours des 3 essais (fig. 2 à 7) fait apparaître des différences importantes entre années. Par contre, ces courbes d'accumulation sont, pour une année donnée, très voisines chez le blé tendre et chez le blé dur ; on note toutefois des accumulations plus importantes chez cette dernière espèce, où les teneurs en proline peuvent atteindre $350 \mu \mathrm{g} . \mathrm{g}^{-1}$ M.F.

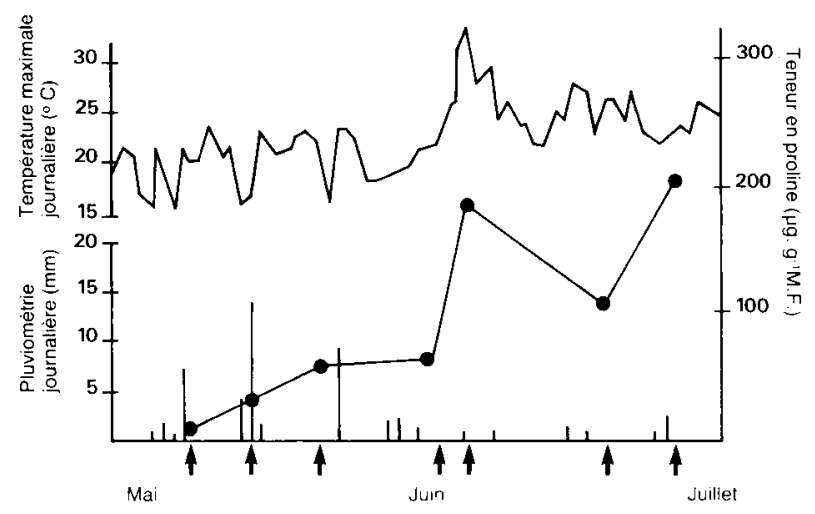

Figure 2

Blé tendre 1980 ; bread wheat 1980.

Données climatiques (pluviométrie et températures maximales journalières) et évolution de la teneur en proline de la dernière feuille au cours du cycle de développement. Moyenne des variétés.

Climatic data (rainfall and maximal daily temperatures) and proline value of the last leaf during the development cycle. Mean of varieties.

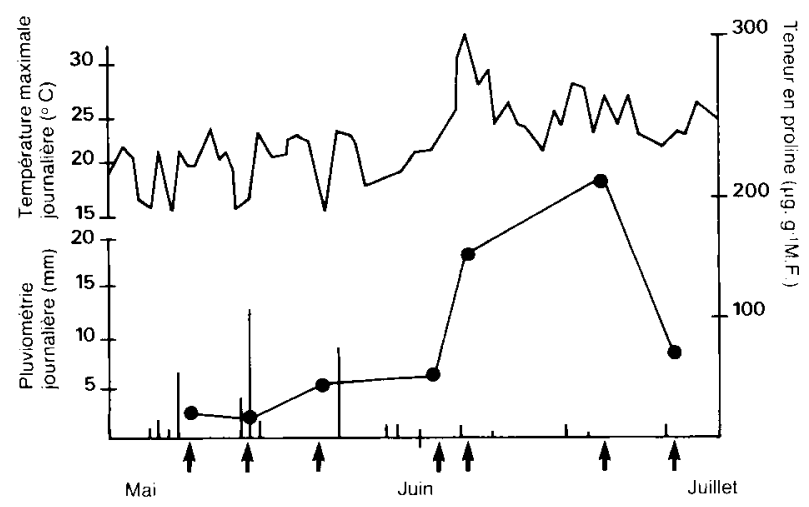

Figure 3

Blé dur 1980 ; durum wheat 1980.

Données climatiques (pluviométrie et températures maximales journalières) et évolution de la teneur en proline de la dernière feuille au cours du cycle de développement. Moyenne des variétés.

Climatic data (rainfall and maximal daily temperatures) and proline value of the last leaf during the development cycle. Mean of varieties.

\section{Essai $n^{\circ} 1(1980)$}

On observe une accumulation importante à partir du $1^{\text {er }}$ juin, date à partir de laquelle les températures maximales journalières sont plus élevées (supérieures à $20^{\circ} \mathrm{C}$ ) et où la pluviométrie est très faible.

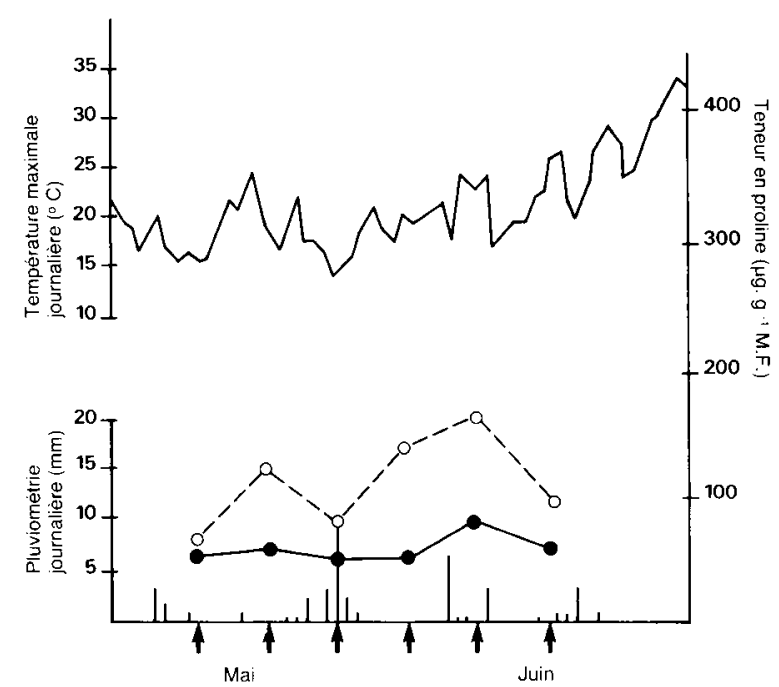

Figure 4

Blé tendre 1981, bread wheat 1981.

Données climatiques (pluviométrie et températures maximales journalières) et évolution de la teneur en proline de la dernière feuille au cours du cycle de développement.

- - moyenne des variétés témoins (cf. tabl. 2). ----o moyenné des variétés testées.

Climatic data (rainfall and maximal daily temperatures) and proline value of the last leaf during the development cycle.

$\longrightarrow$ mean value of the controls (see table 2).

-...-. mean value of all tested varieties.

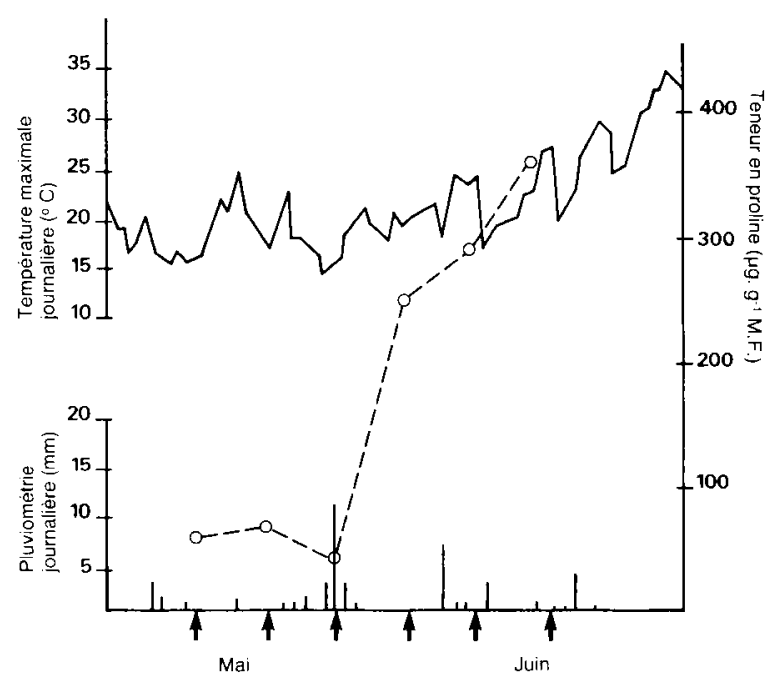

Figure 5

Blé dur 1981; durum wheat 1981.

Données climatiques (pluviométrie et températures maximales journalières) et évolution de la teneur en proline de la dernière feuille au cours du cycle de développement.

Climatic data (rainfall and maximal daily temperatures) and proline value of the last leaf during the development cycle.

o-.-.-. moyenne des variétés testées; mean value of the tested varieties.

\section{Essai $n^{\circ} 2(1981)$}

L'accumulation commence plus tôt (début mai) ; la période fin avril-début mai est caractérisée par l'absence de pluies et des températures élevées (comprises entre 20 et $25^{\circ} \mathrm{C}$ ). La teneur en proline dimi- 


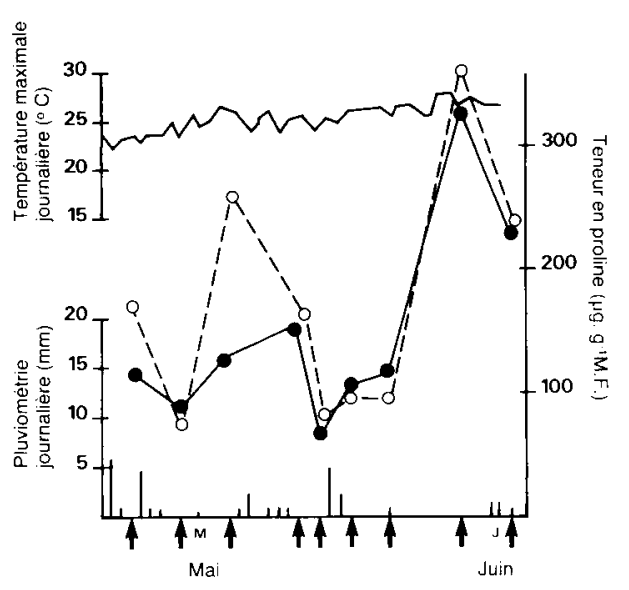

Figure 6

Blé tendre 1983 ; bread wheat 1983.

Données climatiques (pluviométrie et températures maximales journalières) et évolution de la teneur en proline de la dernière feuille au cours du cycle de développement.

-. - moyenne des variétés témoins.

-..---o moyenne des variétés testées.

Climatic data (rainfall and maximal daily temperatures) and proline value of the last leaf during the development cycle.

- - mean value of the controls.

--.---o mean value of all tested varieties.

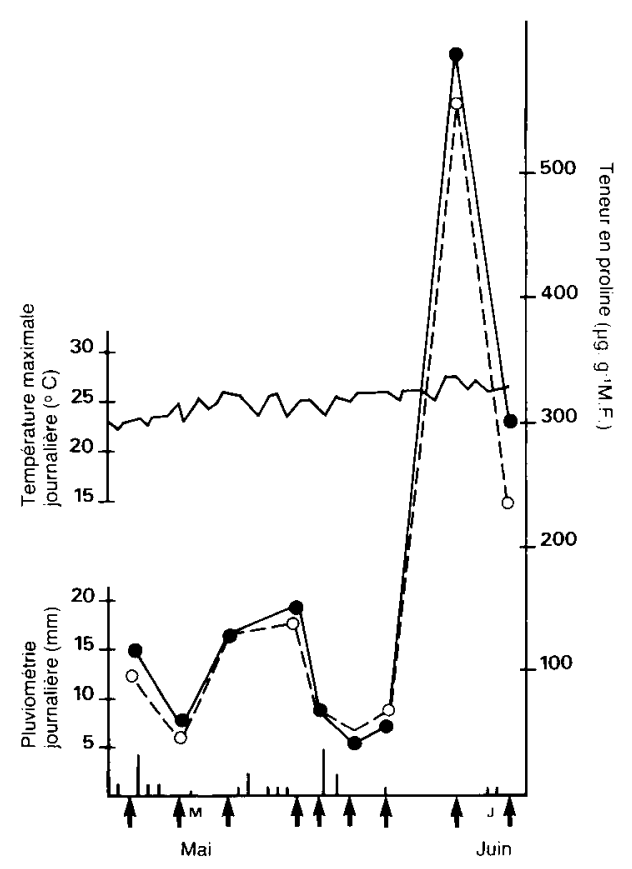

Figure 7

Blé dur 1983 ; durum wheat 1983.

Données climatiques (pluviométrie et températures maximales journalières) et évolution de la teneur en proline de la dernière feuille au cours du cycle de développement.

Climatic data (rainfall and maximal daily temperatures) and proline value of the last leaf during the development cycle.

---.--o moyenne des variétés testées; mean value of the tested varieties.

nue ensuite de façon très marquée jusque vers le 11 mai ; cette diminution de teneur correspond à une période caractérisée par de fortes pluies $(12 \mathrm{~mm}$ le 11 mai) et par des températures basses (inférieures à $15^{\circ} \mathrm{C}$ ). L'accumulation reprend à partir de cette date, pour atteindre début juin, chez le blé dur en particulier des valeurs très élevées : on note, chez le blé tendre des teneurs plus élevées que dans le cas de la moyenne des variétés testées.

\section{Essai $n^{\circ} 3$ (1983)}

On note ici encore une succession de phases d'accumulation et de diminution de la teneur en proline :

- du 22 avril au 29 avril : phase de diminution de la teneur ; la pluviométrie est élevée au cours de cette période $(26 \mathrm{~mm})$;

- du 29 avril au 11 mai : phase d'accumulation ; la pluviométrie est faible $(12 \mathrm{~mm})$ et les températures maximales sont supérieures à celles de la période antérieure ;

- du 11 mai au 16 mai : phase de diminution de la teneur en proline; la pluviométrie est de $14 \mathrm{~mm}$ au cours de ces quelques jours ;

- du 16 mai au 27 mai : phase d'accumulation : la pluviométrie est nulle au cours de cette période et les températures sont en légère augmentation. Les teneurs en proline atteignent des niveaux très élevés (de l'ordre de $190 \mu \mathrm{g} . \mathrm{g}^{-1} \mathrm{M} . \mathrm{F}$. chez le blé tendre, et de $300 \mu \mathrm{g} \cdot \mathrm{g}^{-1} \mathrm{M}$.F. chez le blé dur) ;

- du 27 mai au $1^{\text {er }}$ juin : phase de diminution. On note l'existence d'une légère pluviométrie au cours de cette période.

\section{B. Différences variétales dans l'accumulation de pro- line}

Nous avons, au cours du $3^{\text {e }}$ essai (1983), tenté de classer les variétés selon leur aptitude à accumuler la proline, en prenant par exemple comme référence la teneur maximale obtenue pour une variété donnée, au cours de son cycle. On aboutit aux classements suivants :

\section{Blé tendre}

1) Variétés caractérisées par une forte accumulation :

(205 à $280 \mu \mathrm{g} \cdot \mathrm{g}^{-1}$ M.F.)

Biezostaïa $>$ Cheyenne $>$ Siete Cerros $>$ Mexicano > Mahon Demias > Inia $66 \mathrm{R}>$ Arz.

2) Variétés caractérisées par une accumulation réduite :

(103 à $138 \mu \mathrm{g} . \mathrm{g}^{-1}$ M.F.)

Ducat $>$ Hobbit $>$ Talent $>$ Lutin $>$ Roazon.

3) Variétés « intermédiaires » quant à leur aptitude à accumuler la proline

(150 à $187 \mu \mathrm{g} \cdot \mathrm{g}^{-1}$ M.F.)

Strampelli $>$ Florence-Aurore $>$ Etoile de Choisy $>$ Anza $>$ Top $>$ Champlein $>$ Capitole $>$ Pumaflor.

\section{Blé dur}

Mohamed Ben Bachir $>$ Polonicum $\times$ Zenati Bouteille $>$ Oued Zenati $>$ Bidi $>$ Agathé $>$ Capeiti $>$ Tomclair $>$ Lakota $>$ Durtal $>$ Gloire de Montgolfier $>$ Inrat $69>$ Valnova $>$ Cocorit $>$ Montpellier $>$ Créso > Mondur. 
On voit sur les figures 8 et 9 , l'évolution de la teneur en proline chez les variétés de blé tendre et de blé dur situées aux extrêmes du classement (Hobbitt et Biezostaïa pour le blé tendre, Mohamed Ben Bachir et Mondur pour le blé dur (période 24 mai-30 juin).
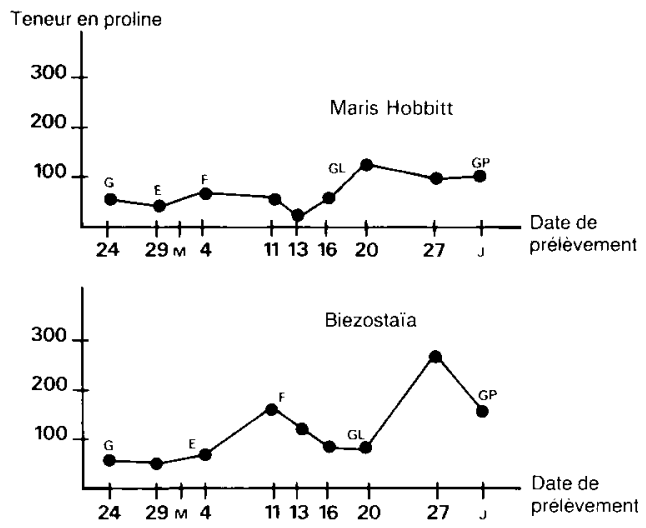

Figure 8

Accumulation de proline chez le blé tendre. Cas des variétés " Maris Hobbitt " (sensible à la sécheresse) et " Biezostaia " (résistante à la sécheresse).

$G=$ gonflement

G.L. = grain laiteux

$E=$ épiaison

G.P. = grain pâteux

$F=$ floraison

Proline accumulation by bread wheat. Case of the varieties 'Maris Hobbitt' (sensitive to drought) and 'Biezostaia' (resistant to drought).
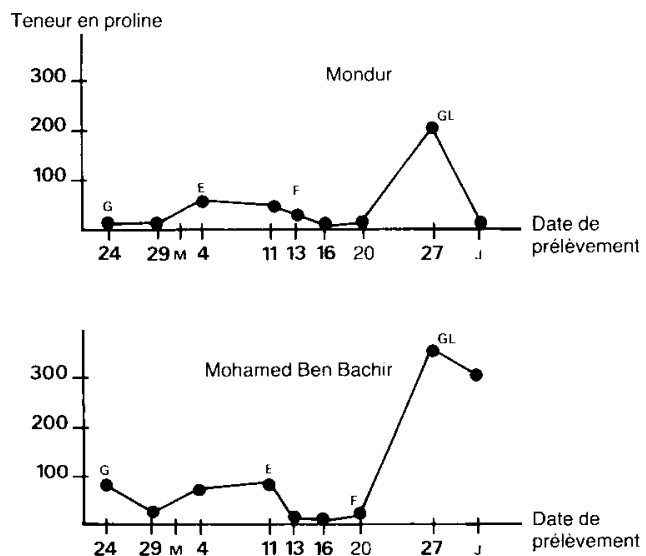

Figure 9

Accumulation de proline chez le blé dur. Cas des variétés "Mondur " (sensible à la sécheresse) et " Mohamed Ben Bachir » (résistante à la sécheresse).

(légende idem fig. 8)

Proline accumulation by durum wheat. Case of the varieties 'Mondur' (sensible to drought) and 'Mohamed Ben Bachir' (resistant to drought).

\section{Relation entre teneur en proline et résistance foliaire à la transpiration}

Le tableau 3 donne les résultats de R.F.T. obtenus lors du palier hydrique et le classement variétal qui peut être opéré sur la base de cette méthode pour les variétés étudiées en 1983 : ce classement est assez proche de celui obtenu à partir des teneurs maximales en proline.

La figure 10 montre la relation qui existe entre la R.F.T. des variétés de blé dur et de blé tendre lors du
TABLEAU 3

Valeur de résistance foliaire à la transpiration, lors du palier hydrique. Classement des variétés de blé tendre et de blé dur.

Foliar resistance values to transpiration. Ranking of bread wheat and durum wheat varieties.

\begin{tabular}{|c|c|c|c|}
\hline Variété & $\begin{array}{l}\text { R.F.T. } \\
\left(\mathrm{s} . \mathrm{cm}^{-1}\right)\end{array}$ & Variété & $\begin{array}{c}\text { R.F.T. } \\
\left(\mathrm{s} . \mathrm{cm}^{-1}\right)\end{array}$ \\
\hline Blé tendre: & & Blé dur: & \\
\hline Biezostaïa & 5,21 & Montpellier & 5,73 \\
\hline Cheyenne & 5,20 & Oued Zenati & 5,75 \\
\hline Mahon Demias & 5,26 & Gloire de Montgolfier & 5,79 \\
\hline Siete Cerros & 5,35 & Bidi 17 & 5,88 \\
\hline Anza & 5,77 & Mohamed Ben Bachir & 6,12 \\
\hline Champlein & 5,96 & Polonicum & 6,43 \\
\hline Arz & 5,97 & Cocorit & 6,61 \\
\hline Capitole & 6,00 & Agathe & 7,13 \\
\hline Mexicano & 6,07 & Tomclair & 7,36 \\
\hline Florence $\times$ Aurore & 6,38 & Inrat 69 & 7,49 \\
\hline Inia $66 \mathrm{R}$ & 6,41 & Durtal & 7,63 \\
\hline Pumaflor & 6,47 & Mondur & 8,08 \\
\hline Maris Hobbitt & 6,58 & Valnova & 8,46 \\
\hline Ducal & 6,69 & Creso & 8,89 \\
\hline Strampelli & 6,78 & Capeiti & 10,64 \\
\hline Lutin & 7,05 & Lakota & 10,73 \\
\hline Talent & 9,12 & & \\
\hline Roazon & 10,60 & & \\
\hline Top & 11,19 & & \\
\hline Etoile de Choisy & 11,59 & & \\
\hline Moyenne & 6,98 & Moyenne & 7,42 \\
\hline
\end{tabular}

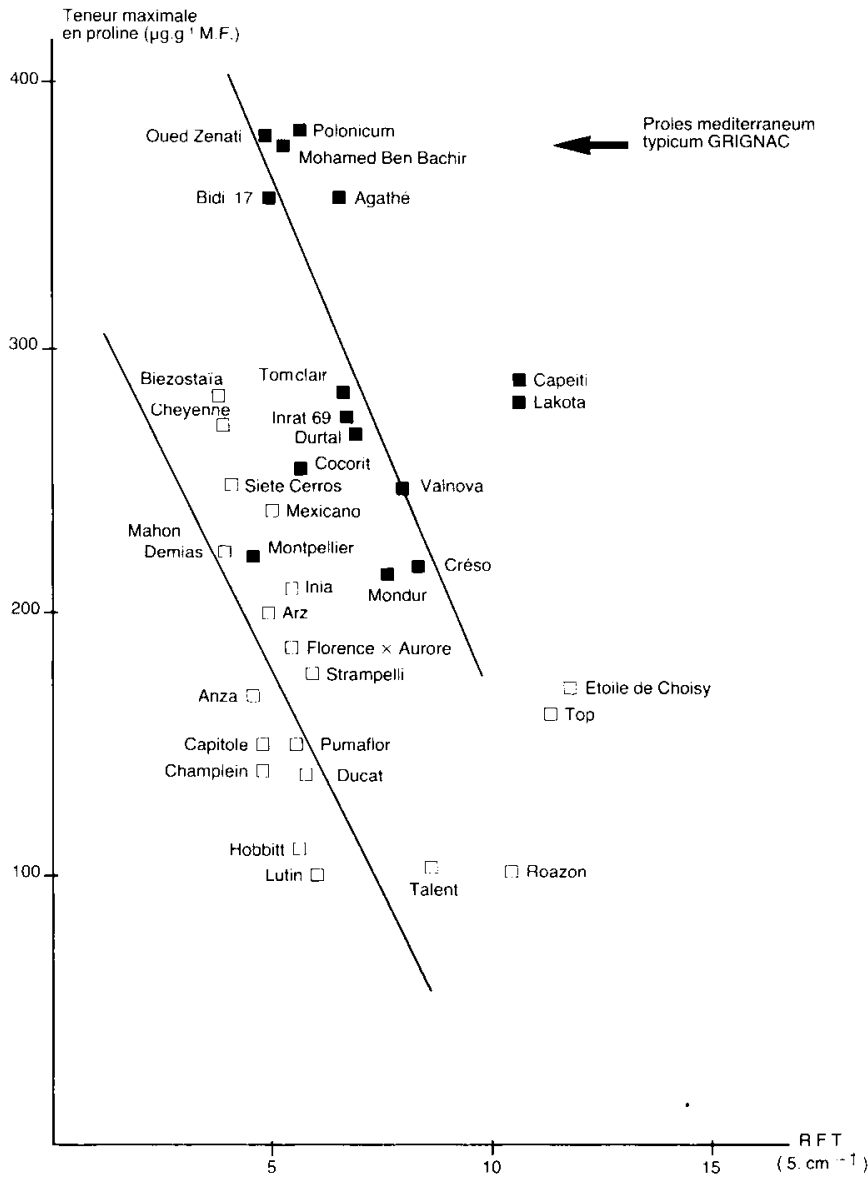

Figure 10

Relation entre la résistance foliaire à la transpiration et la teneur en proline, lors du palier hydrique.

Relation between foliar resistance to transpiration and proline value.

Blé tendre; bread wheat.

Blé dur ; durum wheat. 
palier hydrique et leur teneur en proline à cette date, qui correspond pour la quasi-totalité des variétés à la teneur maximale : les coefficients de corrélation sont de $-0,39$ dans le cas du blé dur, et $-0,51^{*}$ dans le cas du blé tendre.

\section{DISCUSSION}

\section{A. Comparaison générale des essais}

Les différences considérables notées entre les différents essais dans l'allure générale des courbes de teneur en proline suggèrent que l'accumulation de cet amino-acide n'est pas liée aux stades de développement de la plante mais qu'il s'agit d'une réaction de la plante vis-à-vis de certains facteurs du milieu ; le rapprochement qui peut être fait entre l'évolution de la teneur et les données climatiques enregistrées (température maximale journalière, pluviométrie journalière) laisse penser que l'accumulation de la proline dans la feuille est directement liée au déficit hydrique et à l'action des hautes températures. Il serait toutefois nécessaire, afin de préciser l'influence de ces facteurs, de disposer à l'avenir de mesures plus complètes et plus précises concernant le régime hydrique.

Dans le cas du blé tendre, on note, dans les essais $n^{\text {os }} 2$ et 3 , des moyennes d'accumulation plus élevées dans l'ensemble des variétés testées que pour les variétés témoins : ceci nous semble être dû à l'introduction dans l'échantillonnage de variétés originaires d'Afrique du Nord, du Moyen-Orient, ou du Mexique, ayant un niveau de résistance à la sécheresse plus élevé que celui des variétés retenues comme témoins.

\section{B. Différences variétales dans l'accumulation de la proline}

On observe, au cours des 3 essais, des teneurs maximales en proline plus élevées chez les variétés appartenant à l'espèce Triticum durum Desf. ; l'accumulation de l'amino-acide pourrait donc être un des facteurs responsables de la meilleure résistance à la sécheresse notée chez cette espèce.

Le classement des variétés au sein de chaque espèce, établi sur la base de la teneur maximale en proline au cours du cycle, correspond bien à la connaissance empirique que l'on a de la résistance à la sécheresse de ces variétés.

- Chez le blé tendre : on trouve parmi les variétés accumulant de fortes quantités de proline, des variétés dont la résistance au déficit hydrique est connue : variété «steppique» comme «Biezostaïa», variété d'origine nord-africaine, comme "Mahon Demias ", variétés obtenues par le CIMMYT (Centre International d'Amélioration du Maïs et du Blé) comme «Siete Cerros », « Mexicano », « Inia 66 », « Arz ». On trouve parmi les variétés accumulant peu de proline, des variétés originaires de l'Europe temperée océanique : « Roazon », « Champlein », « Top », « Maris Hobbitt ».
- Chez le blé dur: on note l'existence d'un groupe de variétés caractérisées par une teneur en proline élevée. Ce groupe comprend les variétés : «Polonicum », « Oued Zenati », " Mohamed Ben Bachir » et « Bidi 17 » qui sont des variétés originaires d'Afrique du Nord, classées par GRIGNAC (1965) dans le groupe de variétés ou «proles » mediterraneum typicum et connues pour leurs grande résistance à la sécheresse, et la variété «Agathe », issue d'un croisement entre une lignée nord-américaine (Ld 308) et la variété «Montferrier », tirée d'une population d'Afrique du Nord.

Parmi les variétés accumulant peu de proline lors d'un déficit hydrique, on trouve une variété américaine ( Lakota »), une variété comme «Mondur » issue de géniteurs nord-américains (D 5627 «Kubanka » $\times$ «Wells »), des variétés issues de croisements interspécifiques blé dur $\times$ blé tendre comme «Durtal» ou «Tomclair», ainsi que des variétés se rattachant au groupe de variétés ou " proles » mediterraneum italicum (GRIGNAC, 1965), comme « Capeiti » ou «Valnova ».

\section{Relation entre teneur en proline et résistance foliaire à la transpiration}

Il existe chez le blé tendre, une corrélation négative entre teneur maximale en proline et résistance foliaire à la transpiration; le dosage de la proline aboutit à un classement des variétés très voisin de celui obtenu sur la base de la R.F.T. Il faut toutefois signaler le cas des variétés de blé tendre «Top» et «Etoile de Choisy » et des variétés de blé dur « Capeiti » et « Lakota » qui tout en ayant une R.F.T. élevée, accumulent des quantités importantes de proline. Pour une R.F.T. équivalente, les variétés de blé dur ont une teneur maximale en proline plus élevée que les variétés de blé tendre.

\section{CONCLUSION}

L'étude de l'évolution de la teneur en proline au cours du cycle du blé tendre et du blé dur, menée au cours de 3 campagnes, a permis de montrer que l'accumulation de l'amino-acide dans les feuilles est très étroitement liée au manque d'eau et aux hautes températures. Une étude faisant intervenir d'autres mesures du bilan hydrique s'avère toutefois nécessaire pour confirmer et préciser ces résultats.

La variabilité génétique enregistrée pour le caractère " teneur maximale en proline au cours du cycle » a permis un classement des variétés ; les variétés de blé dur ont en général des teneurs plus élevées que les variétés de blé tendre : l'accumulation de proline en cas de déficit hydrique pourrait donc être un des facteurs expliquant la meilleure résistance à la sécheresse du blé dur, au même titre que d'autres facteurs comme la présence de barbes, la présence de stomates nombreux et à fermeture rapide, etc...

Les résultats obtenus au cours de l'essai $n^{\circ} 3$ ont été rapprochés des mesures de R.F.T. lors du palier 
hydrique : les 2 méthodes conduisent à des classements variétaux assez voisins : les variétés de blé tendre ont dans l'ensemble des valeurs de R.F.T. inférieures à celles des variétés de blé dur. Les mesures de teneur en proline et la combinaison de cette mesure avec la mesure de R.F.T. lors du palier hydrique ont permis de mettre nettement en évidence les variétés résistantes à la sécheresse, en particulier chez le blé dur.

L'accumulation de la proline pourrait donc être un véritable mécanisme biochimique de résistance au déficit hydrique, sans qu'il soit toutefois possible en l'état actuel de nos connaissances, de préciser le mode d'action du phénomène. L'évaluation de la capacité d'une variété ou d'une lignée à accumuler de la proline en grandes quantités lors d'un déficit hydrique pourrait donc constituer une méthode intéressante dans la recherche de géniteurs de résistance à la sécheresse.

Reçu le 4 juillet 1984. Accepté le 6 mars 1986.

\section{RÉFÉRENCES BIBLIOGRAPHIQUES}

Bange G. G. J., 1953. On the quantitative explanation of stomatal transpiration. Acta Bot. Neerl., 2, 254-297.

Boggess S. F., Stewart C. R., 1976. Effect of water stress on proline synthesis from radio-active precursors. Plant Physiol., 58, 398-401.

Dreier W., Göring M., 1974. Der Einfluss hoher Salzkonzentrationen auf verschiedene physiologische Parameter von Maiswurzeln. Wiss. Z. der HU. Berlin, Nath. Naturwiss. R., 23, 641-644.

Grignac P., 1965. Contribution à l'étude de Triticum durum Desf. Thèse Doct., Toulouse, $119 \mathrm{p}$.

Hubac C., Guerrier D., 1972. Etude de la composition en acides aminés de deux Carex : le Carex stenophylla Wahl., très résistant à la sécheresse et le Carex setifolia Godion, peu résistant. Effet d'un apport de proline exogène. Oecol. Plant., 7 (2), 147-165.

Lehninger A. L., 1975. Biochemistry, Worth Publ. New York, $1104 \mathrm{p}$.

May L. H., Milthorpe F. L., 1962. Drought resistance of crop plants. Field Crop Abstr., 15 (3), 171-179.

Paquin R., 1977. Effet de basses températures sur la résistance au gel de la luzerne (Medicago media Pers.) et son contenu en proline libre. Physiol. Vég., 15 (4), 657-665.

Parcevaux S. (de), 1963. Transpiration végétale et production de matière sèche. Essai d'interprétation en fonction des facteurs du milieu. Ann. Agron., 14 (5), 655-742.

Parker J., 1972. Water deficit and plant growth, tome 3. Academic Press, New York.

Protsenko D. C., Shmat'koi G., Rubanyuk E. A., 1968. Drought hardiness of winter wheat varieties as related to their amino acid composition. Fiziol. Rast., 15, 680-687.

Sandhu A. S., Laude H. H., 1958. Tests of drought and heat hardiness of winter wheat. Agron. J., 50, 78-81.
Savitskaya N. N., 1967. Problem of accumulation of free proline in barley plant under conditions of soil water deficiency. Fiziol. Rast., 14, 737-739.

Singh T. N., Paleg L. G., Aspinall D., 1973a. Stress metabolism. I : Nitrogen metabolism and growth in barley plant during water stress. Aust. J. Biol. Sci., 26, 45-56.

Singh T. B., Paleg L. G., Aspinall D., 1973b. Stress metabolism. III : Variations in response to water deficit in the barley plant. Aust. J. Biol. Sci., 26, 65-76.

Stewart C. R., Boggess S. F., 1978. Role of carbohydrates in proline accumulation in wilted barley leaves. Plant Physiol., 61, 775778 .

Stewart G. R., Lee J. A., 1974. The role of proline accumulation in halophytes. Planta, 120, 279-289.

Stewart C. R., Boggess S. F., Aspinall D., Paleg L. G., 1977. Inhibition of proline oxidation by water stress. Plant Physiol., 59, 930932.

Stocker D., 1961. Les effets morphologiques et physiologiques du manque d'eau sur les plantes. Recherche sur la zone aride et semiaride. UNESCO, 63-113.

Tall M., Rosental I., 1979. Salt tolerance in Simmondsia chinensis : water balance and accumulation of chloride sodium and proline under low and high salinity. Ann. Bot., 43, 701-708.

Troll W., Lindsley J., 1955. A photometric method for the determination of proline. J. Biol. Chem., 215, 655-660.

Tyankova L. A., 1967. Effects of I.A.A. and 2,4-D on free and bound amino acids in wheat plants recovering after brief drought treatments. Field Crop Abstr., 1531 (20), 3.

Vlasyuk P. A., Shmat'koi G., Rubanyuk E. A., 1968. Role of the trace elements zinc and boron in amino acid metabolism and drought resistance of winter wheat. Fiziol. Rast., 15, 281-287. 